\title{
Combined effects of waggle dance communication and landscape heterogeneity on nectar and pollen uptake in honey bee colonies
}

\author{
Fabian Nürnberger ${ }^{\text {Corresp., }}{ }^{1}$ ， Ingolf Steffan-Dewenter ${ }^{1}$ ， Stephan Härtel ${ }^{1}$ \\ ${ }^{1}$ Department of Animal Ecology and Tropical Biology, Bayerische Julius-Maximilians-Universität Würzburg, Würzburg, Germany \\ Corresponding Author: Fabian Nürnberger \\ Email address: fabian.nuernberger@uni-wuerzburg.de
}

The instructive component of waggle dance communication has been shown to increase resource uptake of Apis mellifera colonies in highly heterogeneous resource environments, but an assessment of its relevance in temperate landscapes with different levels of resource heterogeneity is currently lacking. We hypothesized that the advertisement of resource locations via dance communication would be most relevant in highly heterogeneous landscapes with large spatial variation of floral resources.

To test our hypothesis, we placed 24 Apis mellifera colonies with either disrupted or unimpaired instructive component of dance communication in eight Central European agricultural landscapes that differed in heterogeneity and resource availability. We monitored colony weight change and pollen harvest as measure of foraging success.

Dance disruption did not significantly alter colony weight change, but decreased pollen harvest compared to the communicating colonies by $40 \%$. There was no general effect of resource availability on nectar or pollen foraging success, but the effect of landscape heterogeneity on nectar uptake was stronger when resource availability was high. In contrast to our hypothesis, the effects of disrupted bee communication on nectar and pollen foraging success were not stronger in landscapes with heterogeneous compared to homogenous resource environments.

Our results indicate that in temperate regions intra-colonial communication of resource locations benefits pollen foraging more than nectar foraging, irrespective of landscape heterogeneity. We conclude that the so far largely unexplored role of dance communication in pollen foraging requires further consideration as pollen is a crucial resource for colony development and health. 
1 Title:

2 Combined effects of waggle dance communication and landscape heterogeneity on nectar

3 and pollen uptake in honey bee colonies

4

5 Authors:

Fabian Nürnberger ${ }^{1}$, Ingolf Steffan-Dewenter ${ }^{1}$, Stephan Härtel $^{1}$

7

${ }^{1}$ Department of Animal Ecology and Tropical Biology, Biocenter, University of

Würzburg

10

Würzburg

Germany

Corresponding author:

14

15

16

17

18

19
Fabian Nürnberger ${ }^{1}$

Department of Animal Ecology and Tropical Biology, Biocenter, University of Würzburg

Am Hubland, 97074 Würzburg, Germany

Email address: fabian.nuernberger@uni-wuerzburg.de 


\section{Abstract}

21 The instructive component of waggle dance communication has been shown to increase resource uptake of Apis mellifera colonies in highly heterogeneous resource environments, but an assessment of its relevance in temperate landscapes with different levels of resource heterogeneity is currently lacking. We hypothesized that the advertisement of resource locations via dance communication would be most relevant in highly heterogeneous landscapes with large spatial variation of floral resources. To test our hypothesis, we placed 24 Apis mellifera colonies with either disrupted or unimpaired instructive component of dance communication in eight Central European agricultural landscapes that differed in heterogeneity and resource availability. We monitored colony weight change and pollen harvest as measure of foraging success.

Dance disruption did not significantly alter colony weight change, but decreased pollen harvest compared to the communicating colonies by $40 \%$. There was no general effect of resource availability on nectar or pollen foraging success, but the effect of landscape heterogeneity on nectar uptake was stronger when resource availability was high. In contrast to our hypothesis, the effects of disrupted bee communication on nectar and pollen foraging success were not stronger in landscapes with heterogeneous compared to homogenous resource environments.

Our results indicate that in temperate regions intra-colonial communication of resource locations benefits pollen foraging more than nectar foraging, irrespective of landscape heterogeneity. We conclude that the so far largely unexplored role of dance communication in pollen foraging requires further consideration as pollen is a crucial resource for colony development and health. 


\section{Introduction}

43 Communication is a key feature in social insect colonies, and allows them to allocate the

44 colony's work force effectively to necessary tasks at hand (Hölldobler \& Wilson 2009; Seeley

45 1995; Wilson 1971). An example of this is the recruitment for collaborative foraging, where

46 successful scouts guide idle or unsuccessful nest mates to valuable resource locations

47 (Biesmeijer \& de Vries 2001; Dechaume-Moncharmont et al. 2005; Seeley 1983). The honey bee

48 waggle dance found in the genus Apis is a unique, highly sophisticated and well-studied

49 recruiting behaviour. Honey bees are also capable of spreading information about the resource

50 environment via dance-independent behaviours, e.g. by offering samples of gathered nectar to

51 nest mates via trophallaxis (Farina et al. 2005; Grüter et al. 2006). The waggle dance, however,

52 does not only provide a motivational component that includes information about the presence

53 and identity of rewarding resources. It also includes the well-known instructive component

54 (Menzel et al. 2011; von Frisch 1967). A dancing forager communicates the distance and flight

55 angle relative to the sun's current azimuth, and hence the relatively precise spatial position of a

56 rewarding food source (von Frisch 1967).

57 Studies on the western honey bee (A. mellifera L.) revealed that waggle dances are highly

58 efficient in recruiting foragers to artificial food sources (Sherman \& Visscher 2002; von Frisch

59 1967) and enable honey bee colonies to concentrate their foraging efforts to the most rewarding

60 resources (Schmickl \& Crailsheim 2004; Seeley 1986; Seeley 1995; Seeley et al. 1991).

61 Continuous information exchange about variable resource patches could increase resource uptake

62 rates of honey bee colonies significantly (Donaldson-Matasci \& Dornhaus 2012), and provide

63 fitness advantages (Brown 1988; Dyer 2010; Seeley \& Visscher 1988). Dance communication

64 might also allow for selective pollen foraging by allocating the colony worker force to preferred 
65 pollen sources (Danner et al. 2016), which enables more consistent exploitation of high-quality

66 resources (Donaldson-Matasci \& Dornhaus 2014). Pollen quality may differ significantly

67 between plant species (Haydak 1970), and pollen quality and diversity are important factors for

68 honey bee health (Alaux et al. 2017; Alaux et al. 2010; Di Pasquale et al. 2016; Di Pasquale et al.

69 2013).

70 Nevertheless, the importance of dance communication for an efficient use of nectar or pollen

71 resources in agricultural landscapes is still unclear. Benefits of spatial information conveyed by

72 the instructive component of waggle dancing for colony fitness were found to be highly

73 dependent on resource density, quality and distribution (Donaldson-Matasci \& Dornhaus 2012;

74 Donaldson-Matasci \& Dornhaus 2014; Dornhaus \& Chittka 1999; Dornhaus \& Chittka 2004;

75 Dornhaus et al. 2006; Okada et al. 2012; Sherman \& Visscher 2002). Dornhaus \& Chittka (2004)

76 were able to show a significant effect of dance communication on resource uptake in a complex

77 tropical environment. However, there were no detectable benefits of the instructive component of

78 dance communication in human-modified temperate regions, where distribution of resource

79 patches was less complex (Dornhaus \& Chittka 2004). Within a temperate landscape the benefit

80 of dance communication may change with shifting resource conditions over the seasons

81 (Sherman \& Visscher 2002). Landscapes may differ in the number, proportion and spatial

82 arrangement of different habitat types (Tscharntke et al. 2005), which affects resource

83 distributions. Human-modified temperate landscapes are often dominated by intensively used

84 arable land (Benton et al. 2003; Robinson \& Sutherland 2002). They are characterized by few

85 large habitat patches (Tscharntke et al. 2005), including mass-flowering crops that provide plenty

86 of easily available resources (Holzschuh et al. 2016; Westphal et al. 2003). Such areas have low

87 landscape heterogeneity and form simple resource environments, with easy to find resource 
88 patches. This can reduce the value of instructive information exchange between foragers

89 (Beekman \& Lew 2008). In addition, landscapes may contain varying amounts of semi-natural

90 habitats for which pollen foragers show a strong preference (Danner et al. 2016; Steffan-

91 Dewenter \& Kuhn 2003; Steffan-Dewenter et al. 2002). Semi-natural habitats increase the

92 complexity of a resource environment by generating a more heterogeneous landscape. Here

93 mean patch sizes are more variable and generally smaller (Beekman \& Ratnieks 2000; Steffan-

94 Dewenter et al. 2002). Increased heterogeneity and decreased patch size raise the value of

95 instructive information exchange among honey bee foragers (Beekman \& Lew 2008).

96 Accordingly, the dance frequency of honey bee foragers increases with higher proportion of

97 semi-natural habitats (Steffan-Dewenter and Kuhn 2003).

98 The design of previous studies may have obscured some beneficial effects of waggle dance

99 communication (Schürch \& Grüter 2014). Honey bee colonies in previous studies remained at

100 the same location during the whole experimentation time. This means that foragers were able to

101 gather and exchange information about resources during phases when communication was not

102 disturbed (Sherman and Visscher 2002; Dornhaus and Chittka 2004b; Donaldson-Matasci and

103 Dornhaus 2012; Donaldson-Matasci et al. 2013) and were probably able to profit from this

104 information while dance communication was disrupted.

105 The aim of our study was to investigate the importance of the information about resource

106 locations conveyed by honey bee dance communication for nectar and pollen foraging success of

107 colonies exposed to landscapes with varying resource heterogeneity. We experimentally

108 disrupted the instructive component of dance communication in honey bee colonies and

109 measured nectar and pollen uptake rates. Unlike previous studies, we performed this in a number

110 of spatially separated human-modified temperate landscapes featuring a variety of levels of 
111 complexity and resource availability. For the first time in this context, we used landscape

112 heterogeneity, i.e. heterogeneity in the spatial arrangement of resource patches within a

113 landscape, which describes the complexity of the resource environment on the landscape level

114 and independently from the amount of available resources. We expected that the value of dance

115 communication for colony performance would increase with decreasing resource availability and

116 increasing landscape heterogeneity.

117

118 Material and Methods

119 Study region

120 The study was conducted in Central Europe, in the vicinity of Würzburg, Germany. Within the

121 study region, simple landscapes, dominated by intensive agriculture, and complex landscapes

122 with a mixture of arable land, woodland, hedgerows, meadows and settlements can be

123 delineated. In order to assess the role of waggle dance communication in different resource

124 environments we selected eight circular landscapes (distances among landscapes ranged from 5.0

125 to $31.2 \mathrm{~km}$ ) with differing proportions of intensively used arable land and semi-natural habitats

126 (Tab. 1). Landscapes were analysed within a radius of $2 \mathrm{~km}$ (1265.64 ha area), because mean bee

127 foraging distances under comparable circumstances were shown to lie well within this range

128 (Steffan-Dewenter \& Kuhn 2003), and more than 90\% of pollen foraging recruitments advertise

129 patches within this distance to the colony (Danner et al. 2014). The experiment took place in late

130 summer $2013\left(18^{\text {th }}\right.$ July $-18^{\text {th }}$ August 2013).

131

132 Landscape-level floral resource availability and heterogeneity 
133 Resource availability in each of the eight study landscapes was assessed in two steps. Firstly, we

134 distinguished between habitats that provided noteworthy plant resources for honey bees and

135 those that were unlikely to be utilized for foraging. Resource providing habitats were hedgerows,

136 intensively or extensively used grassland, fallows, meadow orchards, maize fields, sunflower

137 fields, legume fields (including alfalfa, white and red clover and legume mixtures) and non-

138 flowering crop fields (predominantly weeds in beet and cereal fields and vineyards). The relative

139 cover of each habitat type was computed using a geographical information system (Arc-GIS) and

140 digital land use data, which was validated by field inspections. Secondly, we estimated total

141 flower cover on the $2000 \mathrm{~m}$ scale. For this purpose, flower cover was assessed in at least three

142 randomly selected $100 \mathrm{~m}^{2}$ plots in each habitat type that provided measurable amounts of

143 resources (Scheper et al. 2015). Total flower cover was extrapolated by summing estimations of

144 mean flower cover per area multiplied by the relative cover of each habitat type across all habitat

145 types in each landscape. In order to keep track of changes in resource distributions over time, the

146 assessment of the flower cover was done twice. The two discrete timespans for which flower

147 cover was assessed in this study were named period A and period B. Period A lasted from $17^{\text {th }}$

148 July to $2^{\text {nd }}$ August 2013, while vegetation period B lasted from $3^{\text {rd }}$ August to $18^{\text {th }}$ August 2013.

149 Mean patch size of resource-providing habitats, a configurational measure of landscape

150 complexity, was used as proxy for resource heterogeneity in the landscape. Heterogeneous and

151 more complex resource environments are characterized by small mean patch sizes. Flower cover

152 (resource availability) and mean patch size (landscape heterogeneity) were not correlated

153 significantly $(r=-0.30 ; t=-1.16, d f=14, p=0.265)$.

154

155 Study organism 
156 Twenty-four colonies of Apis mellifera carnica were established on $11^{\text {th }}$ July 2013 by making

157 nucleus colonies that were equal in size. Each colony was provided with three fully occupied

158 brood combs, two food combs (Zander measure) and a mated queen. All queens were sister-

159 queens from a professional breeder (Schüler, Münster, Germany). Nucleus colonies were

160 inserted into hive boxes with nine frames. The empty space was filled with two empty combs

161 and two wax sheet frames. Sets of three honey bee colonies were placed in the centre of each

162 study landscape on individual levelled tables.

163

\section{Disruption of waggle dance communication}

165 The hive box design enabled us to disrupt the instructive component of waggle dance

166 communication using a method following the established approach of Sherman \& Visscher

167 (2002) and Dornhaus \& Chittka (2004). The hive boxes were placed on levelled tables, and

168 rotation of hives by $90^{\circ}$ allowed for combs to be positioned horizontally, preventing bees from

169 orienting their dances in a specific angle to the gravitational cue. All incoming foragers in rotated

170 hives were forced to enter the hive box via the top frame next to the window, to encourage them

171 to dance there (Dornhaus \& Chittka 2004). Combs were held in place by a tight-fitting slot

172 system that prevented tilting while hive boxes were rotated. In a dark hive without additional

173 cues, dances are performed in random directions and no longer provide consistent spatial

174 information about resource locations (Dornhaus \& Chittka 2004; Sherman \& Visscher 2002; von

175 Frisch 1967). The successful disruption of waggle dance orientation on horizontal combs in our

176 experimental hive boxes was confirmed by in-hive video recordings (Fig. S1). Dance orientation

177 on horizontal combs can be re-established if dancers are allowed to see the sun, blue sky or any

178 directional light source (Sherman \& Visscher 2002; von Frisch 1967). As an additional treatment 
179 we attempted to restore dance orientation by providing a directional light source in form of a 180 closable circular window of $2.5 \mathrm{~cm}$ in diameter. However, dance observations revealed that

181 dance orientation could not be fully restored (Fig S1). Therefore, we do not report results of this 182 treatment.

183 We analysed groups of colonies with (1) disrupted communication: combs were positioned 184 horizontally and dances were disoriented, in order to investigate the impact of disrupted dance 185 communication; and (2) intact communication: combs were positioned vertically in a dark hive, 186 allowing for unimpaired dances.

187 All sets of three colonies were moved between the eight landscapes every fourth day during night time. This was repeated seven times, so that each set of colonies was placed in each of the eight landscapes for four days by the end of the experiment. For the statistical analysis each fourday period was regarded as a distinct time step. All communication treatments were randomly reassigned to the three colonies in each landscape at each time step. All 24 colonies were tested in each treatment and landscape. Minimum distance between consecutive colony locations was ten kilometres to prevent foragers from returning to former colony sites (mean $=19.2 \mathrm{~km}, S D= \pm 6.9$ $\mathrm{km})$. At the same time, this procedure reduced the value of information about the resource environment that was previously acquired by foragers. This prevented carry-over effects from masking the influence of waggle dance communication on resource uptake. The spatial arrangement of resource patches and of landmarks that could guide workers during foraging flights differed considerably between landscapes. Foragers were shown to perform waggle dances advertising resource patches in up to $4.4 \mathrm{~km}$ distance on the first day after moving to a new environment (Danner et al. 2014). Due to the methodology, it was not possible to record data on colonies blindly. 


\section{Colony development}

204 The presence of the marked queen and brood in the colonies was confirmed every eight days and 205 the total brood area was estimated. In one colony a queen had to be replaced by a reserve sister 206 queen, because she died in the course of the experiment. Data obtained from this colony were not

207 excluded from the presented models, as excluding data did not significantly change model 208 outcomes.

\section{Colony weight}

Colony weight change is supposed to reflect resource uptake on colony level. Nectar is the main

212 factor influencing colony weight changes on a daily basis (Meikle et al. 2008; Seeley 1995). A portable platform balance (Kern EOB35K10) was used to weigh the colonies. Each colony was weighed at the beginning of the experiment and on the first and fourth day at each site. Weighing took place during night time, when all foragers were back in their nest and there was no further 216 resource uptake.

\section{Pollen uptake}

219 The complete pollen forage of each colony was sampled throughout the first day that colonies spent in a new landscape. A total of 192 pollen samples was collected. The pollen was gathered

221 using pollen traps with removable perforated plates ( $5 \mathrm{~mm}$ diameter holes) in front of the colony entrance (Keller et al. 2005). Pollen traps were activated during night time after moving the colonies. Deactivation and pollen collection occurred during the consecutive night, following the

224 weighing of colonies. 
225 The pollen samples were stored in a $-20^{\circ} \mathrm{C}$ freezer. Later on, pollen samples were vacuum-dried,

226 cleaned from insect parts and other artefacts and weighed to the nearest $0.01 \mathrm{~g}$ using a lab scale

227 (Kern Type 430-33). Mean weight-loss ( $\pm s e$ ) by vacuum drying was $17.5 \% \pm 1.3 \%$.

\section{Statistics}

230 We used linear mixed-effects models in R version 3.2.0 (R Core Team 2015) with the package

231 lme4 (Bates et al. 2014) to test for effects of instructive dance communication, flower cover and

232 mean patch size, as well as respective interactions, on colony weight change and dry-weight of

233 pollen harvest. Effects on colony weight change were only tested during times when pollen traps

234 were not active. Identity of colony, site and time step were included as random factors in each

235 model to address pseudo-replication and design imbalances. $P$-values, degrees of freedom and $F$ -

236 values were obtained using the R-package lmerTest using the Satterthwaite approximation for

237 degrees of freedom (Kuznetsova et al. 2015). Minimum adequate models were identified using

238 ANOVA-tests. $P$-values of factors that were not included in the minimum adequate models but

239 that were relevant for the hypotheses were calculated by adding the respective factor to the

240 minimum adequate model. As integral part of the main hypothesis, the effects of communication

241 treatments on dependent variables were always shown in the figures, regardless of statistical

242 significance. Data on pollen dry-weight were cubic-root transformed to meet the assumption of

243 normal distribution for linear models. Model residuals were visually inspected for spatial

244 autocorrelation and violation of assumptions of normality and homoscedasticity. See Tab. 2 for

245 an overview of tested factors and interactions.

246

247 Results 
249 Mean daily colony weight changes did not differ significantly between colonies with disrupted or

250 intact dance communication (Fig. 1; see Tab. 2 for statistics). Due to the scarcity of floral

251 resources in late summer in the region of Lower Franconia, Germany, all colonies lost weight

252 over the study period and during most time steps (mean weight change $=-37.87 \mathrm{~g} / \mathrm{day}$, se $=$

$253 \pm 6.04$ g/day, $n=192$; Fig. 1).

254 Rotating the combs to a horizontal position and thereby disrupting dance communication

255 significantly reduced the dry-weight of pollen harvest by $40.25 \%$ (Fig. 2; Tab. 2).

Effects of flower cover and mean patch size

258

259

260

261

262

263

264

265

266

267

268

269

270

Mean flower cover of habitat types ranged from $0-85.2 \%$ (mean $=8.4 \%$; se $= \pm 1.6 \%)$. The flower cover in the studied landscapes varied considerably, both among the eight landscapes and between the two distinct mapping periods (Tab.1). In every landscape and during each mapping period we recorded highly rewarding patches of nectar-providing crops like sunflower and legume fields, flower-rich areas promoted by agri-environmental schemes, or flower-rich grasslands. Overall there was no significant effect of flower cover on colony weight change (Fig 3; Tab. 2) or dry-weight of pollen harvest (Fig. 4; Tab. 2).

We used mean patch size in a landscape to define landscape heterogeneity (see Tab.1 for patch size range), with higher mean patch size in landscapes with lower heterogeneity. Mean patch size was significantly positively correlated with colony weight change (Fig. 5; Tab. 2) and dry-weight of pollen harvest (Fig. 6; Tab. 2). Additionally, flower cover affected the impact of mean patch size on colony weight change, with stronger effects of mean patch size when flower cover was high (Fig. 7; Tab. 2). 
271 There was no significant interaction between flower cover or mean patch size of the studied

272 landscapes and the effect of dance communication on foraging success (Fig. 3 - 6; Tab. 2).

273

274 Discussion

275 In this study we analysed the interplay between the instructive component of dance

276 communication and landscape structure, with regard to colony foraging success. Contrary to our

277 hypothesis, we found that honey bee communication about locations of rewarding floral

278 resources did not promote the nectar intake of bee colonies in temperate agricultural landscapes.

279 The amount of pollen collected in colonies within hives that were rotated in order to disrupt

280 dance orientation was reduced by $40 \%$, indicating an important role of instructive

281 communication in pollen foraging. Our data reveal that the amount of brood reared by a colony

282 which is a main driver of pollen foraging activity was not affected by hive rotations (Fig S2) but

283 we cannot exclude that the horizontal comb position has further unknown effects on brood

284 rearing behaviour or pollen foraging and storage. Landscape heterogeneity affected nectar and

285 pollen foraging success, but in contrast to our expectation, the benefits of instructive dance

286 communication were not modulated by the complexity of the resource environment. Resource

287 availability within the tested landscapes had no direct effects on nectar or pollen foraging

288 success, but altered effects of landscape heterogeneity on nectar foraging success.

289 It is important to keep in mind that we, and others, disrupted only the instructive information in

290 waggle dance recruitment behaviour. Waggle dances also include information about the presence

291 of rewarding nectar or pollen sources, as well as about their identity (von Frisch 1967; von

292 Frisch 1968). Dancing foragers are also known to activate idle foragers as well as to reactivate

293 experienced but currently unemployed foragers (Grüter \& Farina 2009), so that dancing 
294 generally increases forager recruitment (Gilley 2014; von Frisch 1968). Thus dancing can have a

295 positive effect on resource uptake rates that is unrelated to communication of resource location

296 directions.

297 In our study, colony weight change was not impacted by manipulation of dance communication,

298 although we deliberately placed colonies in experimentally selected environments where

299 effective communication should offer advantages for foraging success. We tested a number of

300 different landscapes that varied significantly in resource availability and heterogeneity. The

301 study was conducted during late summer, when resources in the study region were generally

302 scarce and colonies lost in weight, but some resource-rich patches were still available and

303 information exchange was expected to be valuable (Okada et al. 2012; Sherman \& Visscher

304 2002). Additionally, repeatedly moving the colonies to a new environment created an

305 exceptionally short-living resource environment. This forced foragers to repeatedly update

306 information about locations of profitable resources instead of making good use of previously

307 acquired information, which might have masked effects in earlier experiments (Schürch \&

308 Grüter 2014). In contrast to our hypothesis the high temporal turnover and the spatial

309 heterogeneity of resource patches experienced by foragers did not increase the importance of

310 communication. In temperate landscapes the instructive component of waggle dance

311 communication might only prove to be advantageous for nectar foraging in environments under

312 very specific conditions, like strong intra- or interspecific competition (Donaldson-Matasci \&

313 Dornhaus 2012; Seeley \& Visscher 1988) or during specific seasonal resource distributions

314 (Sherman \& Visscher 2002). While the conditions were deliberately chosen in order to identify

315 the specific conditions under which communication of resource location would be beneficial, it is

316 important to note that these conditions are not representative for the whole flowering period. In 
317 early spring, for example, resources would also be scarce but possibly much more patchily

318 distributed in form of few flowering trees and scrubs, which may increase the value of

319 directional dance communication. Additionally, if instructive dance communication does only

320 outweigh dancing costs if advertised resources can be used over extended time periods (Schürch

$321 \&$ Grüter 2014), repeatedly moving colonies every four days prevented us from identifying these

322 long-term benefits. This should be addressed in future field experiments. Contrary to our

323 findings in nectar foraging, our data show that the disruption of instructive dance communication

324 had a strong negative effect on pollen foraging. To our knowledge, only two related studies also

325 investigated the effect of instructive dance communication directly on pollen forage instead of

326 colony weight change (Donaldson-Matasci \& Dornhaus 2012; Donaldson-Matasci \& Dornhaus

327 2014). However, the studies were restricted to Sonoran Desert scrub and grassland habitats. In

328 these non-temperate landscapes dance communication increased pollen uptake rates

329 independently of resource availability, but only if resource distribution was patchy. Additionally,

330 instructive dance communication also proved to be advantageous, depending on resource

331 conditions (Donaldson-Matasci \& Dornhaus 2012). Due to the study design foragers could make

332 use of information on resource locations gathered before communication was disrupted (Schürch

333 \& Grüter 2014) or ignore available dance information in favour of previously acquired

334 information on resource locations (Grüter \& Ratnieks 2011). Therefore, these studies possibly

335 failed to reveal the actual extent of the effect of dance communication. As colonies in our study

336 were moved to unknown landscapes with considerably different spatial features at the same time

337 at which treatments in individual colonies were changed, we prevented that foragers profited

338 from previously acquired information on resource locations. This allowed us to assess the total

339 benefits of directional dance information under the given conditions. Our findings for temperate 
340 landscapes under the conditions of sub-optimal resource availability do not support the

341 hypothesis that resource distribution affects the value of directed dance communication in honey

342 bee colonies. It remains to be confirmed if this is also true when foragers can profit from the

343 directional dance information for a longer period of time, as we only investigated effects on

344 short-term benefits. The fact that in the tested temperate landscapes dance communication

345 always improved pollen foraging, but never nectar foraging, is remarkable. We suspect that this

346 is related to the circumstance that honey bee colonies exploit a higher diversity of plant species

347 for pollen than for nectar (Requier et al. 2015). The identity and diversity of pollen sources may

348 have a strong effect on colony health (Alaux et al. 2010; Di Pasquale et al. 2016; Di Pasquale et

349 al. 2013). Dance communication may allow for a selective and diverse but still effective pollen

350 foraging, but may be less important for effective nectar foraging in temperate landscapes. In fact,

351 it was shown that waggle dance communication affects the composition of pollen forage

352 (Donaldson-Matasci \& Dornhaus 2014). A mechanistic explanation for the differences in our

353 findings between nectar and pollen foraging might be, that pollen foragers are more motivated to

354 follow dances and make use of the instructive component of the dances, e.g. of scouts that

355 advertise novel resource patches. It has been shown that previous experience in the field and in-

356 hive olfactory information affect the way foragers deal with available dance information (Farina

357 et al. 2012). In addition, pollen foragers were shown to have a preference for pollen collected

358 from plant species found in semi-natural habitats (Danner et al. 2016) which are generally

359 relatively small, scarce, patchily distributed and probably quickly depleted. Therefore pollen

360 foragers could profit more from the instructive component of dance communication than nectar

361 foragers that commonly forage in presumably more easy to find mass-flowering crop fields or

362 other floral resources with abundant nectar supply (Beekman \& Lew 2008). Additionally, pollen 
363 advertisement in plants can be more limited in time than nectar advertisement and pollen within

364 inflorescences can be rapidly depleted (Herrera 1990; Stone et al. 1999). High ephemerality of

365 pollen sources and possibly increased competition would increase the benefits of effective

366 communication (Dornhaus \& Chittka 2004; Seeley \& Visscher 1988). We cannot rule out the

367 possibility that additional factors affected pollen foraging activity, as disrupting dance

368 communication coincided with hive rotation (Sherman \& Visscher 2002). It could be argued that

369 rotating the hives affected the brood, brood-provisioning behaviour or brood rearing activity

370 which is known to be strongly correlated with pollen foraging activity (Al-Tikrity et al. 1972;

371 Dreller \& Tarpy 2000; Free 1967; Pankiw et al. 1998). While a small proportion of larvae may

372 be malformed, brood rearing in general and egg-laying activity of queens are not known to be

373 affected by horizontal comb position (Chauvin 1960). In our study the amount of reared brood

374 seemed unaffected by comb position (Fig S2). We cannot exclude that other components of

375 brood rearing activity are affected by hive rotation and further research on this might help to

376 confirm that indeed disruption of the instructive dance communication caused the observed

377 effects on pollen foraging. However, the random exchange of treatments every four days

378 combined with a considerably longer development time of bee brood minimised possible effects

379 of comb rotation on brood rearing. Although our treatment to control for effects of hive rotation

380 by restoring directed dances on horizontal combs did not work, we therefore conclude that the

381 measured effects of comb orientation on pollen foraging success were most probably due to the

382 disrupted instructive dance information. Incoming pollen was only sampled during the first day

383 within a new environment, in order not to disrupt protein supply and hence brood rearing. To

384 which extent our findings can be extrapolated to longer time periods needs further investigation. 
385 Studies on landscape-related foraging patterns of honey bee colonies are still rare (Couvillon et

386 al. 2014; Danner et al. 2014; Danner et al. 2016; Härtel \& Steffan-Dewenter 2014). In our study,

387 variation in the generally low resource availability within late summer in temperate landscapes

388 had no direct effect on foraging success. Irrespective of overall resource availability, foragers

389 probably concentrated their efforts on few but most valuable resource patches. However,

390 especially in the most resource rich landscapes, landscape heterogeneity had a strong effect on

391 foraging success. Foraging was most successful in landscapes that contained flower-rich, large

392 and easy to find resource patches, like mass-flowering crop fields. With increasing landscape

393 heterogeneity, i.e. decreasing patch sizes, colony foraging success decreased. Foragers

394 presumably spent less time within the smaller, quickly depleted patches (Cresswell \& Osborne

395 2004) and hence probably more time on travelling between the scattered patches. This may

396 reduce foraging efficiency (Westphal et al. 2006).

397

398 Conclusions

399 Although there is an increasing number of theoretical studies and field experiments addressing

400 the possible benefits of the instructive component of waggle dance communication (Beekman \&

401 Lew 2008; Donaldson-Matasci et al. 2013; Donaldson-Matasci \& Dornhaus 2012; Donaldson-

402 Matasci \& Dornhaus 2014; Dornhaus \& Chittka 2004; Dornhaus et al. 2006; Okada et al. 2012;

403 Schürch \& Grüter 2014; Sherman \& Visscher 2002), this study demonstrates that we still lack

404 some essential knowledge regarding its actual relevance on colony level. Even in heterogeneous

405 temperate landscapes and under specific conditions that were expected to increase the benefits of

406 advertisement of resource locations, there were no short-term benefits of instructive dance

407 communication for nectar foraging. In an unknown environment individual search abilities of 
408 honey bee foragers and newly established knowledge of resource locations may be sufficient to

409 secure colony foraging success. It is possible that communicating nectar resource locations in

410 temperate landscapes will only provide benefits on the long-term (Schürch \& Grüter 2014),

411 which was prevented in our study. Importantly, our data indicate that, within temperate

412 landscapes, waggle dancing plays a far more important role in pollen foraging than in nectar

413 foraging. As pollen is the major protein source in honey bee hives, dance communication can be 414 expected to have significant effects on colony development and health. This underpins the 415 potential evolutionary advantage of dance communication and suggests that future research 416 should focus more on pollen foraging ecology of honey bees.

\section{Acknowledgements}

419 We would like to thank Stefan Berg from the Bayerische Landesanstalt für Weinbau und 420 Gartenbau, Veitshöchheim, for his expert support and provision of honey bee colonies for the 421 experiment, Susanne Schiele for her excellent assistance during the fieldwork, and Patrick Kohl

422 for recording waggle dance runs. We thank Emily Poppenborg, Sarah Redlich, and the reviewers 423 for valuable comments on the manuscript. The Bundesamt für Kartographie und Geodäsie kindly 424 provided access to land use data on the study site.

\section{References}

427 Al-Tikrity W, Benton A, Hillman R, and Clarke Jr W. 1972. The relationship between the amount of unsealed brood in honeybee colonies and their pollen collection. Journal of Apicultural Research 11:9-12. 10.1080/00218839.1972.11099693 
430 Alaux C, Allier F, Decourtye A, Odoux JF, Tamic T, Chabirand M, Delestra E, Decugis F, Le

431

432

433

434

435

436

437

438

439

440

441

442

443

444

445

446

447

448

449

450

Conte Y, and Henry M. 2017. A 'Landscape physiology' approach for assessing bee health highlights the benefits of floral landscape enrichment and semi-natural habitats. Scientific Reports 7:10. 10.1038/srep40568

Alaux C, Ducloz F, Crauser D, and Le Conte Y. 2010. Diet effects on honeybee immunocompetence. Biology Letters. 10.1098/rsb1.2009.0986

Bates D, Maechler M, Bolker B, and Walker S. 2014. lme4: Linear mixed-effects models using Eigen and S4. R package version 1.1-7.

Beekman M, and Lew JB. 2008. Foraging in honeybees - when does it pay to dance? Behavioral Ecology 19:255-262. 10.1093/beheco/arm117

Beekman M, and Ratnieks F. 2000. Long-range foraging by the honey-bee, Apis mellifera L. Functional Ecology 14:490-496. 10.1046/j.1365-2435.2000.00443.x

Benton TG, Vickery JA, and Wilson JD. 2003. Farmland biodiversity: is habitat heterogeneity the key? Trends in Ecology \& Evolution 18:182-188. 10.1016/S0169-5347(03)00011-9

Biesmeijer JC, and de Vries H. 2001. Exploration and exploitation of food sources by social insect colonies: a revision of the scout-recruit concept. Behavioral Ecology and Sociobiology 49:89-99. 10.1007/s002650000289

Brown CR. 1988. Enhanced Foraging Efficiency Through Information Centers: A Benefit of Coloniality in Cliff Swallows. Ecology 69:602-613. 10.2307/1941009

Chauvin R. 1960. Sur les possibilités d'adaptation chez les insectes sociaux et spécialement chez l'abeille. Insectes Sociaux 7:101-108. 10.1007/bf02224074 
451 Couvillon Margaret J, Schürch R, and Ratnieks Francis LW. 2014. Dancing Bees Communicate

452 a Foraging Preference for Rural Lands in High-Level Agri-Environment Schemes.

453 Current Biology 24:1212-1215. 10.1016/j.cub.2014.03.072

454 Cresswell JE, and Osborne JL. 2004. The effect of patch size and separation on bumblebee 455 456 foraging in oilseed rape: implications for gene flow. Journal of Applied Ecology 41:539546. 10.1111/j.0021-8901.2004.00912.x

457

458

459

460

461

462

463

464

465

466

467

468

469

470

471

472

Danner N, Härtel S, and Steffan-Dewenter I. 2014. Maize pollen foraging by honey bees in relation to crop area and landscape context. Basic and Applied Ecology 15:677-684. 10.1016/j.baae.2014.08.010

Danner N, Molitor AM, Schiele S, Härtel S, and Steffan-Dewenter I. 2016. Season and landscape composition affect pollen foraging distances and habitat use of honey bees. Ecological Applications 26:1920-1929. 10.1890/15-1840.1

Dechaume-Moncharmont FX, Dornhaus A, Houston AI, McNamara JM, Collins EJ, and Franks NR. 2005. The hidden cost of information in collective foraging. Proceedings of the Royal Society Biological Sciences Series B 272:1689-1695. 10.1098/rspb.2005.3137

Di Pasquale G, Alaux C, Le Conte Y, Odoux J-F, Pioz M, Vaissière BE, Belzunces LP, and Decourtye A. 2016. Variations in the Availability of Pollen Resources Affect Honey Bee Health. PLOS ONE 11:e0162818. 10.1371/journal.pone.0162818

Di Pasquale G, Salignon M, Le Conte Y, Belzunces LP, Decourtye A, Kretzschmar A, Suchail S, Brunet J-L, and Alaux C. 2013. Influence of pollen nutrition on honey bee health: do pollen quality and diversity matter? PLOS ONE 8:e72016.

10.1371/journal.pone.0072016 
473 Donaldson-Matasci MC, DeGrandi-Hoffman G, and Dornhaus A. 2013. Bigger is better:

474 honeybee colonies as distributed information-gathering systems. Animal Behaviour

475 85:585-592. 10.1016/j.anbehav.2012.12.020

476

477

478

479

480

481

482

483

484

485

486

487

488

489

490

491

492

493

494

495

Donaldson-Matasci MC, and Dornhaus A. 2012. How habitat affects the benefits of communication in collectively foraging honey bees. Behavioral Ecology and Sociobiology 66:583-592. 10.1007/s00265-011-1306-z

Donaldson-Matasci MC, and Dornhaus A. 2014. Dance Communication Affects Consistency, but Not Breadth, of Resource Use in Pollen-Foraging Honey Bees. PLOS ONE 9. 10.1371/journal.pone.0107527

Dornhaus A, and Chittka L. 1999. Insect behaviour - Evolutionary origins of bee dances. Nature 401:38-38. 10.1038/43372

Dornhaus A, and Chittka L. 2004. Why do honey bees dance? Behavioral Ecology and Sociobiology 55:395-401. 10.1007/s00265-003-0726-9

Dornhaus A, Klugl F, Oechslein C, Puppe F, and Chittka L. 2006. Benefits of recruitment in honey bees: effects of ecology and colony size in an individual-based model. Behavioral Ecology 17:336-344. 10.1093/beheco/arj036

Dreller C, and Tarpy DR. 2000. Perception of the pollen need by foragers in a honeybee colony. Animal Behaviour 59:91-96. 10.1006/anbe.1999.1303

Dyer FC. 2010. The dance Language. Oxford: Academic Press.

Farina WM, Grüter C, and Arenas A. 2012. Olfactory Information Transfer During Recruitment in Honey Bees. In: Galizia CG, Eisenhardt D, and Giurfa M, eds. Honeybee Neurobiology and Behavior: A Tribute to Randolf Menzel. Dordrecht: Springer Netherlands, 89-101. 
496 Farina WM, Grüter C, and Díaz PC. 2005. Social learning of floral odours inside the honeybee 497 hive. Proceedings of the Royal Society B: Biological Sciences 272:1923.

498 Free JB. 1967. Factors determining the collection of pollen by honeybee foragers. Animal 499 Behaviour 15:134-144. 10.1016/S0003-3472(67)80024-1

500 Gilley DC. 2014. Hydrocarbons Emitted by Waggle-Dancing Honey Bees Increase Forager 501 Recruitment by Stimulating Dancing. PLOS ONE 9. 10.1371/journal.pone.0105671 502 Grüter C, Acosta LE, and Farina WM. 2006. Propagation of olfactory information within the 503 504 honeybee hive. Behavioral Ecology and Sociobiology 60:707-715. 10.1007/s00265-006-

Grüter C, and Ratnieks FLW. 2011. Honeybee foragers increase the use of waggle dance information when private information becomes unrewarding. Animal Behaviour 81:949954. 10.1016/j.anbehav.2011.01.014

Härtel S, and Steffan-Dewenter I. 2014. Ecology: Honey Bee Foraging in Human-Modified Landscapes. Current Biology 24:R524-R526. 10.1016/j.cub.2014.04.052

Haydak MH. 1970. Honey bee nutrition. Annual Review of Entomology 15:143-156. 10.1146/annurev.en.15.010170.001043

514 Herrera CM. 1990. Daily Patterns of Pollinator Activity, Differential Pollinating Effectiveness, and Floral Resource Availability, in a Summer-Flowering Mediterranean Shrub. Oikos 58:277-288. 10.2307/3545218

517 Hölldobler B, and Wilson EO. 2009. The superorganism: the beauty, elegance, and strangeness $518 \quad$ of insect societies: WW Norton \& Company. 
519 Holzschuh A, Dainese M, González-Varo JP, Mudri-Stojnić S, Riedinger V, Rundlöf M, Scheper

520 J, Wickens JB, Wickens VJ, and Bommarco R. 2016. Mass-flowering crops dilute

521 pollinator abundance in agricultural landscapes across Europe. Ecology Letters 19:1228-

$522 \quad 1236.10 .1111 /$ ele. 12657

523 Keller I, Fluri P, and Imdorf A. 2005. Pollen nutrition and colony development in honey bees-

524 Part II. Bee World 86:27-34. 10.1080/0005772X.2005.11099650

525 Kuznetsova A, Brockhoff PB, and Christensen RHB. 2015. lmerTest: tests in linear mixed

526 effects models. R package version 2.0-20.

527 Meikle WG, Rector BG, Mercadier G, and Holst N. 2008. Within-day variation in continuous

528 hive weight data as a measure of honey bee colony activity. Apidologie 39:694-707.

$529 \quad 10.1051 /$ apido:2008055

530 Menzel R, Kirbach A, Haass W-D, Fischer B, Fuchs J, Koblofsky M, Lehmann K, Reiter L,

531 Meyer H, Nguyen H, Jones S, Norton P, and Greggers U. 2011. A Common Frame of

532 Reference for Learned and Communicated Vectors in Honeybee Navigation. Current

$533 \quad$ Biology 21:645-650. 10.1016/j.cub.2011.02.039

534 Okada R, Akamatsu T, Iwata K, Ikeno H, Kimura T, Ohashi M, Aonuma H, and Ito E. 2012.

535 Waggle dance effect: dancing in autumn reduces the mass loss of a honeybee colony.

$536 \quad$ Journal of Experimental Biology 215:1633-1641. 10.1242/jeb.068650

537 Pankiw T, Page Jr RE, and Kim Fondrk M. 1998. Brood pheromone stimulates pollen foraging

538 in honey bees (Apis mellifera). Behavioral Ecology and Sociobiology 44:193-198.

$539 \quad 10.1007 / \mathrm{s} 002650050531$

540 R Core Team. 2015. R: A language and environment for statistical computing. Vienna, Austria:

541 R Foundation for Statistical Computing. 
542 Requier F, Odoux J-F, Tamic T, Moreau N, Henry M, Decourtye A, and Bretagnolle V. 2015.

543 Honey bee diet in intensive farmland habitats reveals an unexpectedly high flower

544 richness and a major role of weeds. Ecological Applications 25:881-890. 10.1890/14-

$545 \quad 1011.1$

546 Robinson RA, and Sutherland WJ. 2002. Post-war changes in arable farming and biodiversity in

547 Great Britain. Journal of Applied Ecology 39:157-176. 10.1046/j.1365-

$548 \quad 2664.2002 .00695 . x$

549 Scheper J, Bommarco R, Holzschuh A, Potts SG, Riedinger V, Roberts SP, Rundlöf M, Smith

550 HG, Steffan-Dewenter I, and Wickens JB. 2015. Local and landscape-level floral

551 resources explain effects of wildflower strips on wild bees across four European

$552 \quad$ countries. Journal of Applied Ecology 52:1165-1175. 10.1111/1365-2664.12479

553 Schmickl T, and Crailsheim K. 2004. Costs of environmental fluctuations and benefits of

554 dynamic decentralized foraging decisions in honey bees. Adaptive Behavior 12:263-277.

$555 \quad 10.1177 / 105971230401200311$

556 Schürch R, and Grüter C. 2014. Dancing Bees Improve Colony Foraging Success as Long-Term

557 Benefits Outweigh Short-Term Costs. PLOS ONE 9. 10.1371/journal.pone.0104660

558 Seeley TD. 1983. Division of labor between scouts and recruits in honeybee foraging.

559 Behavioral Ecology and Sociobiology 12:253-259. 10.1007/BF00290778

560 Seeley TD. 1986. Social Foraging by Honeybees - How Colonies allocate Foragers among

561 Patches of Flowers. Behavioral Ecology and Sociobiology 19:343-354.

562

$10.1007 / \mathrm{bf00295707}$

563 Seeley TD. 1995. The wisdom of the hive: The social physiology of honey bee colonies:

564 Cambridge, MA: Harvard University Press. 
565 Seeley TD, Camazine S, and Sneyd J. 1991. Collective decision-making in honey bees - how

566 colonies choose among nectar sources. Behavioral Ecology and Sociobiology 28:277-

$567 \quad 290.10 .1007 / \mathrm{BF} 00175101$

568 Seeley TD, and Visscher PK. 1988. Assessing the benefits of cooperation in honeybee foraging -

569 search costs, forage quality, and competitive ability. Behavioral Ecology and

$570 \quad$ Sociobiology 22:229-237. 10.1007/bf00299837

571 Sherman G, and Visscher PK. 2002. Honeybee colonies achieve fitness through dancing. Nature

572 419:920-922. 10.1038/nature01127

573 Steffan-Dewenter I, and Kuhn A. 2003. Honeybee foraging in differentially structured

574 landscapes. Proceedings of the Royal Society Biological Sciences Series B 270:569-575.

575 10.1098/rspb.2002.2292

576 Steffan-Dewenter I, Munzenberg U, Burger C, Thies C, and Tscharntke T. 2002. Scale577 dependent effects of landscape context on three pollinator guilds. Ecology 83:1421-1432.

Stone GN, Gilbert F, Willmer P, Potts S, Semida F, and Zalat S. 1999. Windows of opportunity and the temporal structuring of foraging activity in a desert solitary bee. Ecological Entomology 24:208-221. 10.1046/j.1365-2311.1999.00181.x

Tscharntke T, Klein AM, Kruess A, Steffan-Dewenter I, and Thies C. 2005. Landscape perspectives on agricultural intensification and biodiversity-ecosystem service management. Ecology Letters 8:857-874. 10.1111/j.1461-0248.2005.00782.x University Press. 
587 von Frisch K. 1968. The role of dances in recruiting bees to familiar sites. Animal Behaviour $588 \quad 16: 531-533.10 .1016 / 0003-3472(68) 90047-x$

589 Westphal C, Steffan-Dewenter I, and Tscharntke T. 2003. Mass flowering crops enhance 590 pollinator densities at a landscape scale. Ecology Letters 6:961-965. 10.1046/j.1461$591 \quad 0248.2003 .00523 . x$

592 Westphal C, Steffan-Dewenter I, and Tscharntke T. 2006. Foraging trip duration of bumblebees 593 in relation to landscape-wide resource availability. Ecological Entomology 31:389-394. $594 \quad 10.1111 / \mathrm{j} .1365-2311.2006 .00801 . \mathrm{x}$

595 Wilson EO. 1971. The insect societies. Cambridge, MA: Harvard University Press. 


\section{Table $\mathbf{1}$ (on next page)}

Landscape parameters of the eight selected landscapes for a 2000 m radius buffer around experimental colonies.

Flower cover is given for the two distinct mapping periods. Means \pm standard errors and ranges. 


\begin{tabular}{lll}
\hline Landscape parameter & Mean \pm se & Range \\
\hline \% Semi-natural habitat & $7.8 \pm 2.5$ & $0.4-16.6$ \\
\% Arable land & $71.5 \pm 5.7$ & $51.1-89.6$ \\
Flower cover - Period A [ha] & $23.9 \pm 2.9$ & $11.1-33.3$ \\
Flower cover - Period B [ha] & $12.5 \pm 3.2$ & $3.4-26.0$ \\
Mean patch size [ha] & $1.5 \pm 0.2$ & $0.8-2.3$ \\
\hline
\end{tabular}

1 


\section{Table 2 (on next page)}

Results of linear mixed effects models relating colony weight change and dry-weight of pollen harvest to explanatory variables.

$n=8$ landscapes, $n=24$ colonies, $n=127$ colony weight measurements, $n=127$ pollen samples. 


\begin{tabular}{|c|c|c|c|c|}
\hline Explanatory variables & $\mathrm{nDF}$ & dDF & $F$ & $p$ \\
\hline \multicolumn{5}{|l|}{ Colony weight change $[\mathrm{g}]$} \\
\hline Communication & 1 & 111.93 & 1.03 & 0.312 \\
\hline Flower cover & 1 & 125.84 & 2.64 & 0.107 \\
\hline Mean patch size & 1 & 8.93 & 10.35 & 0.011 \\
\hline Communication $\times$ flower cover & 1 & 111.37 & 0.40 & 0.528 \\
\hline Communication $\times$ mean patch size & 1 & 111.43 & 0.01 & 0.924 \\
\hline Flower cover $\times$ mean patch size & 1 & 114.93 & 7.25 & $<0.001$ \\
\hline Communication $\times$ flower cover $\times$ mean patch & 1 & 111.11 & 0.39 & 0.532 \\
\hline \multicolumn{5}{|l|}{ Dry-weight of pollen harvest [g] } \\
\hline Communication & 1 & 111.99 & 11.02 & 0.001 \\
\hline Flower cover & 1 & 107.25 & 0.52 & 0.473 \\
\hline Mean patch size & 1 & 7.816 & 8.18 & 0.022 \\
\hline Communication $\times$ flower cover & 1 & 110.75 & 0.001 & 0.977 \\
\hline Communication $\times$ mean patch size & 1 & 110.32 & 1.28 & 0.260 \\
\hline Flower cover $\times$ mean patch size & 1 & 97.57 & 1.96 & 0.164 \\
\hline Communication $\times$ flower cover $\times$ mean patch & 1 & 110.72 & 1.17 & 0.282 \\
\hline
\end{tabular}




\section{Figure 1}

Effects of dance communication on mean daily weight change ( \pm se) of honey bee colonies.

Disrupted: colonies with horizontal comb position and disoriented dances; and intact: colonies with non-affected dance communication on vertically positioned combs. ns: $p>$ 0.05 .

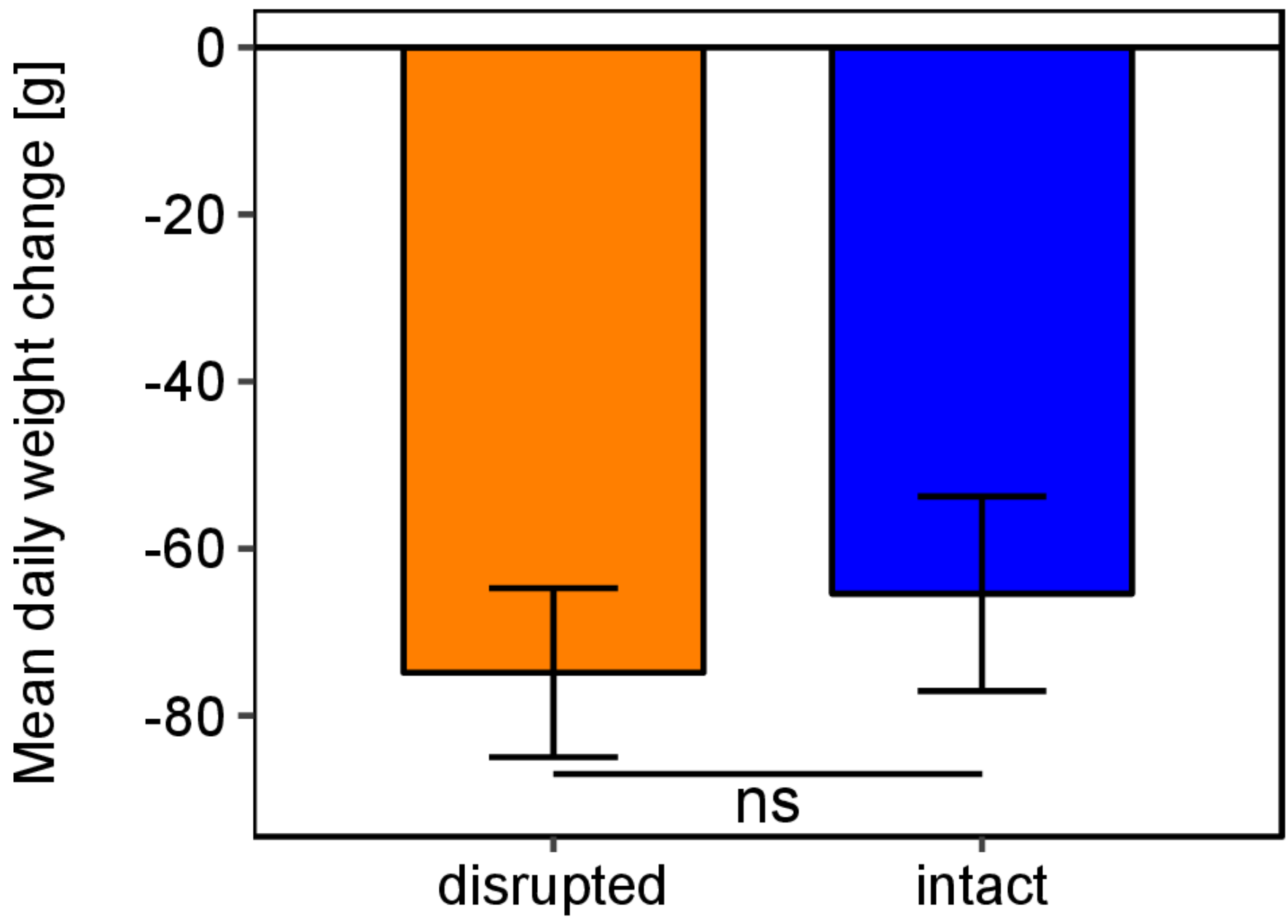

Communication 


\section{Figure 2}

Effects of dance communication on mean dry-weight $( \pm s e)$ of pollen harvest collected by honey bee colonies.

For treatments see Fig. 1. ${ }^{* * *}: p \leq 0.001$.

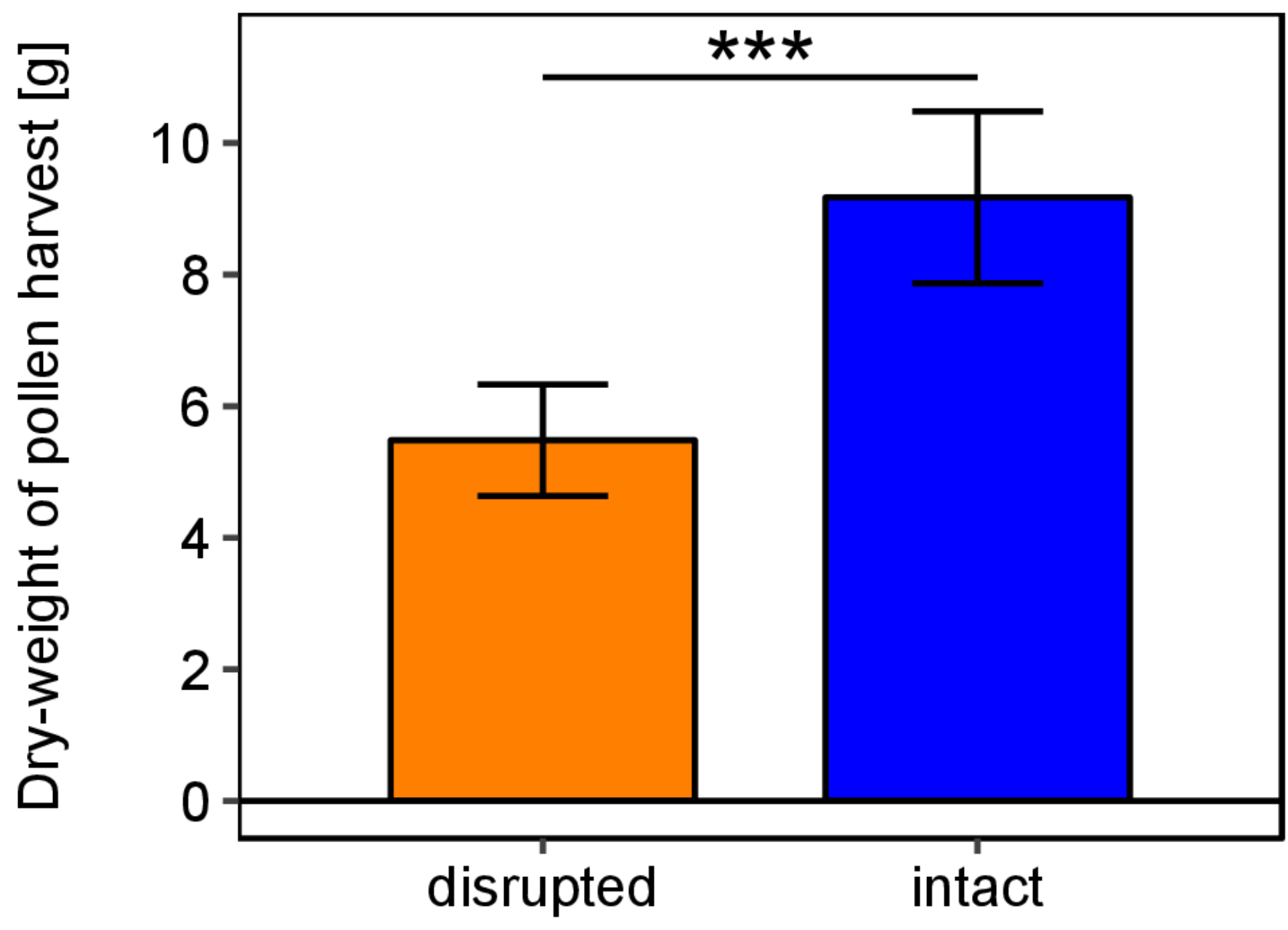

Communication 
Figure 3

The relationship between flower cover and mean daily weight change ( $\pm s e)$ of honey bee colonies.

For statistics see Tab. 2.

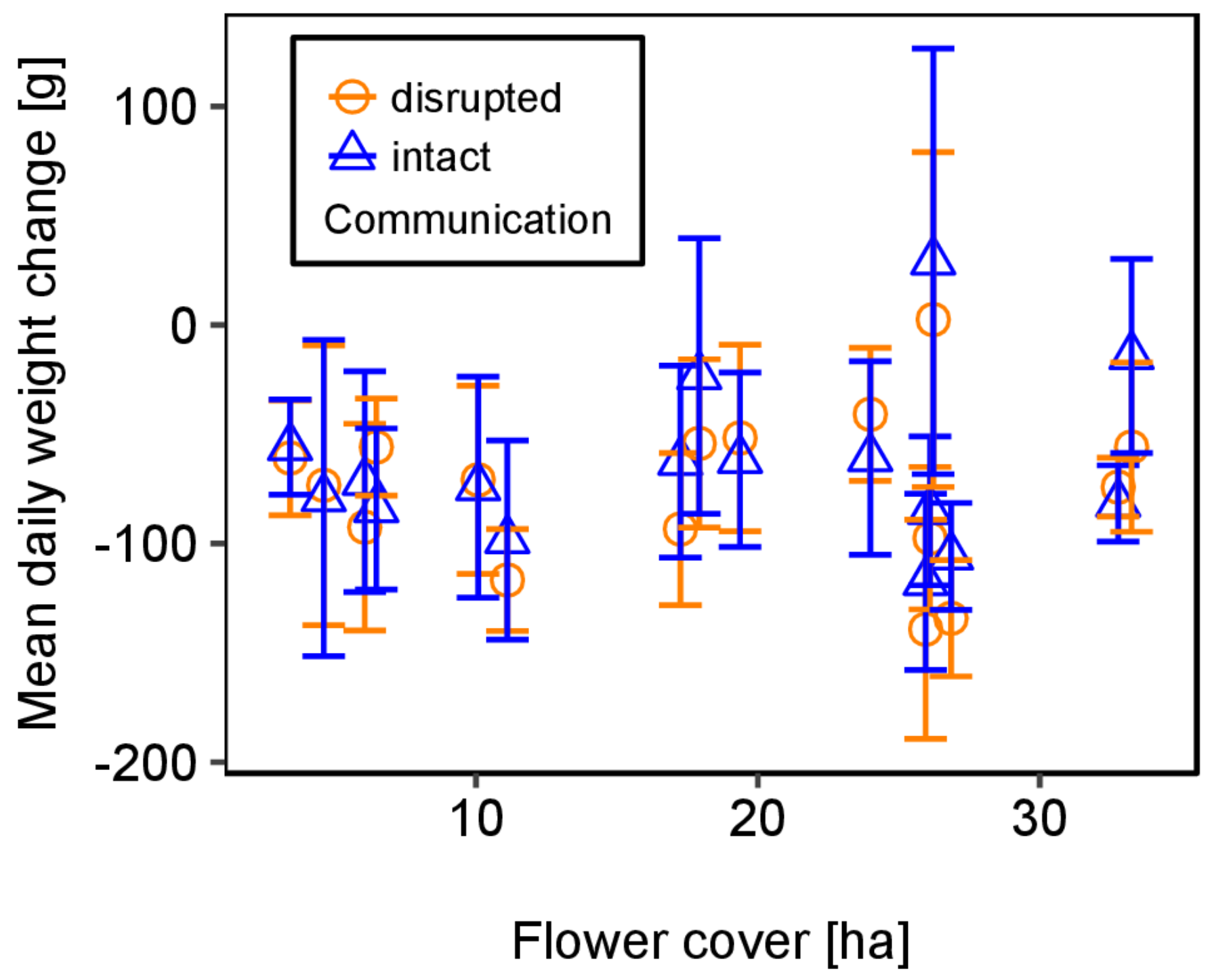


Figure 4

The relationship between flower cover and mean dry-weight ( $\pm s e$ ) of pollen collected by honey bee colonies.

For statistics see Tab. 2.

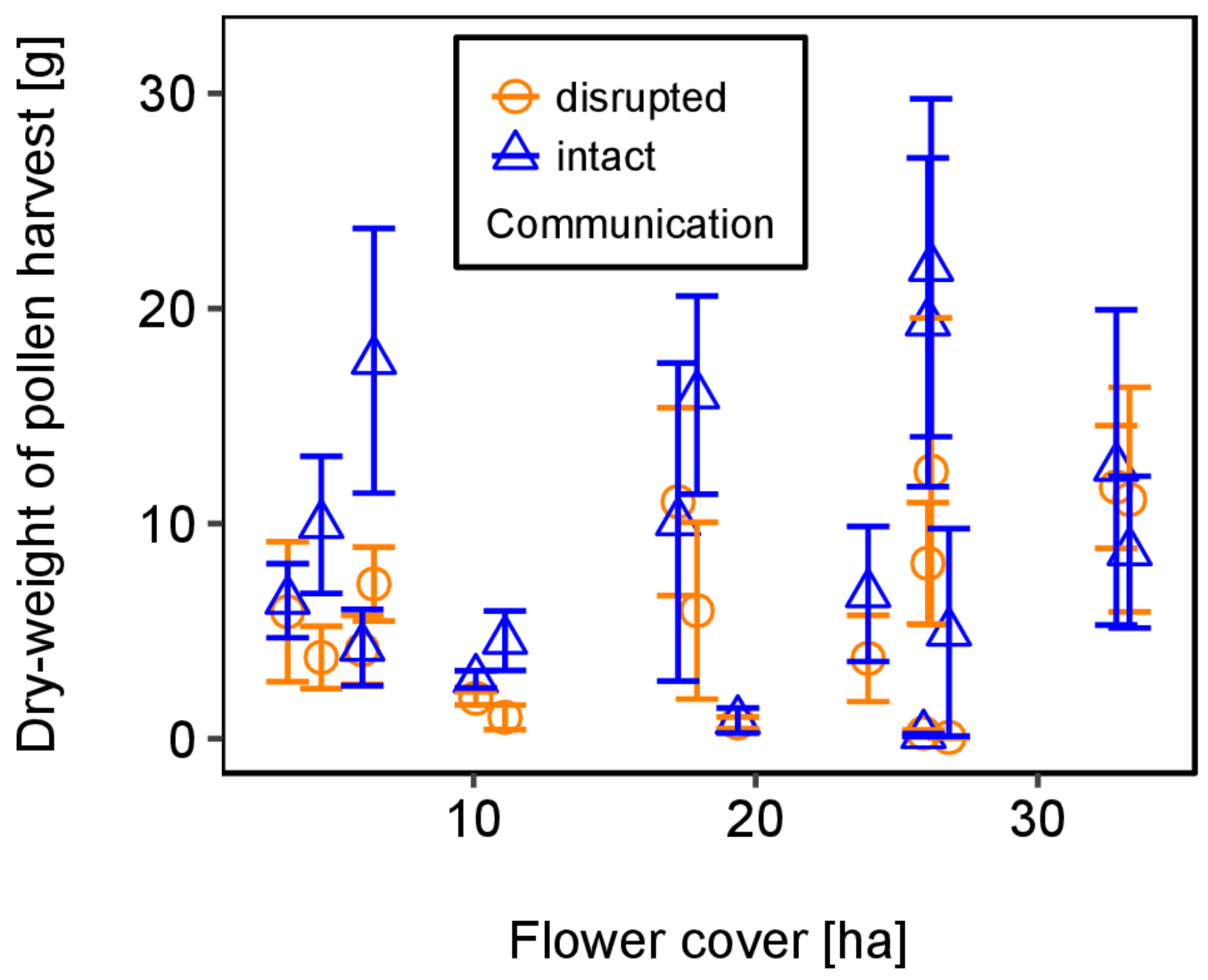


Figure 5

The relationship between mean patch size and mean daily weight change $( \pm s e)$ of honey bee colonies.

Regression line fitted with linear model. For statistics see Tab. 2.

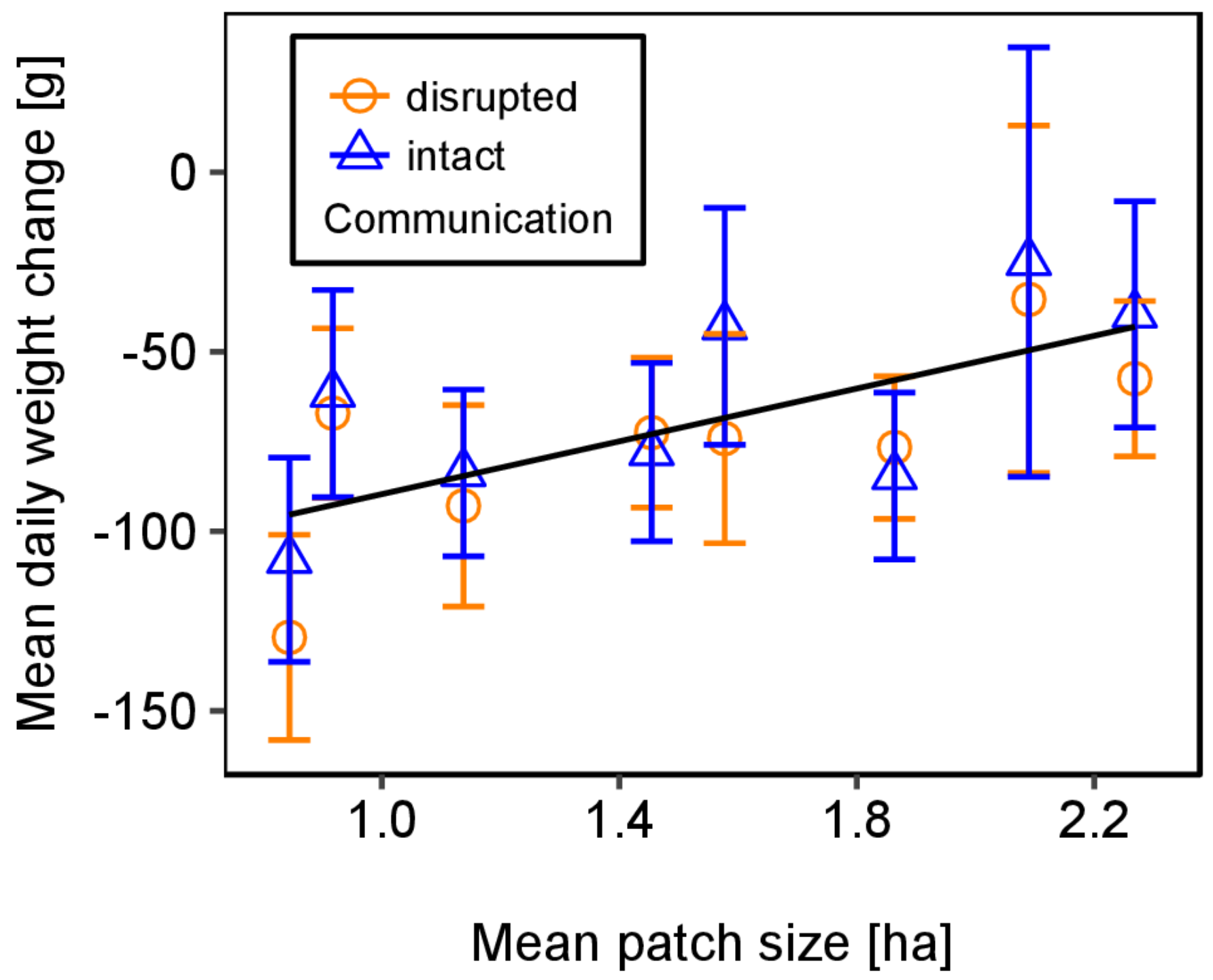


Figure 6

The effect of mean patch size within landscapes on mean dry-weight ( \pm se) of pollen collected by honey bee colonies.

Regression line fitted with linear model. For statistics see Tab. 2.

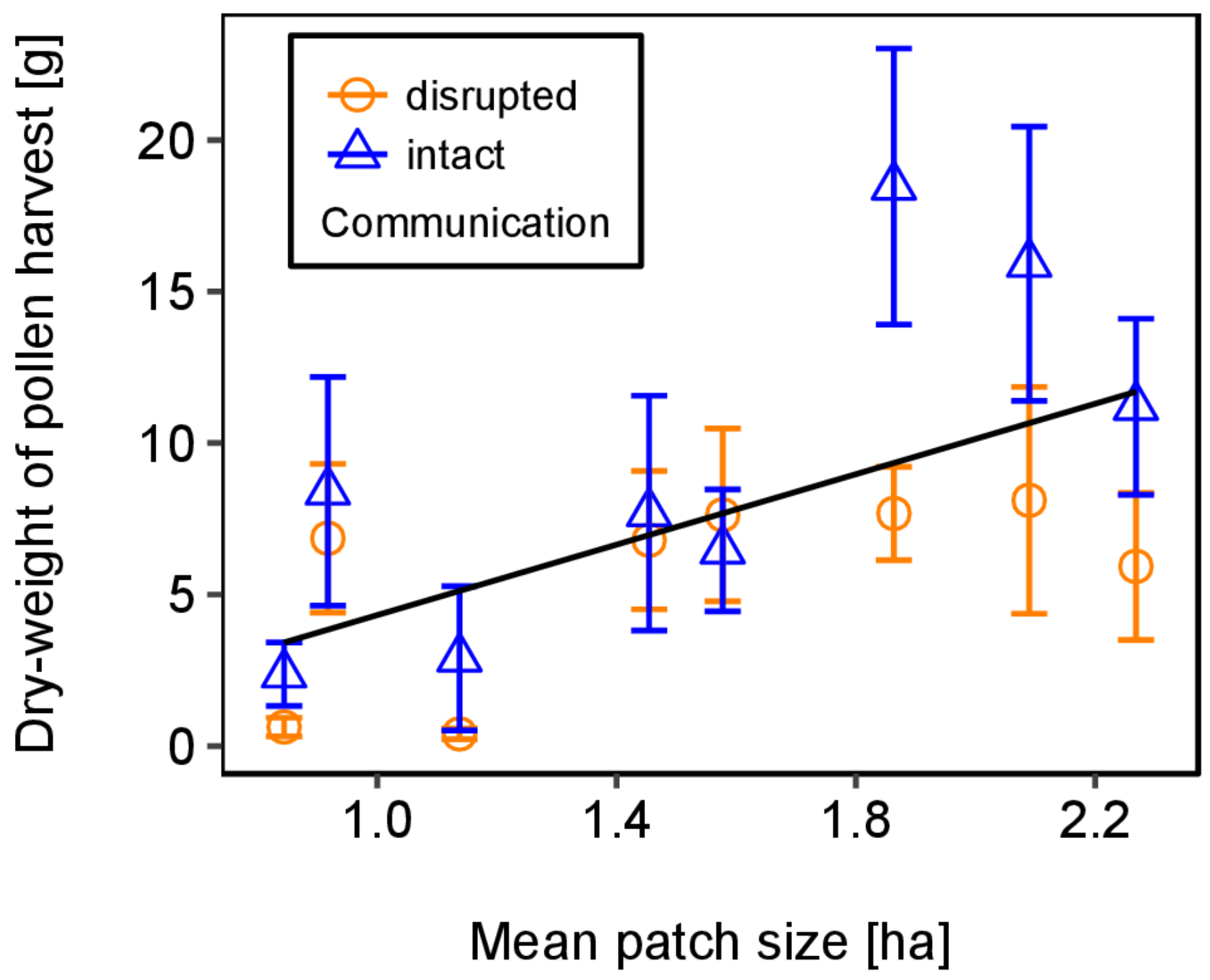


Figure 7

The effect of mean patch size on mean daily weight change of honey bee colonies depending on flower cover within the landscape.

Grey area: 95\% confidence interval. For statistics see Tab. 2.

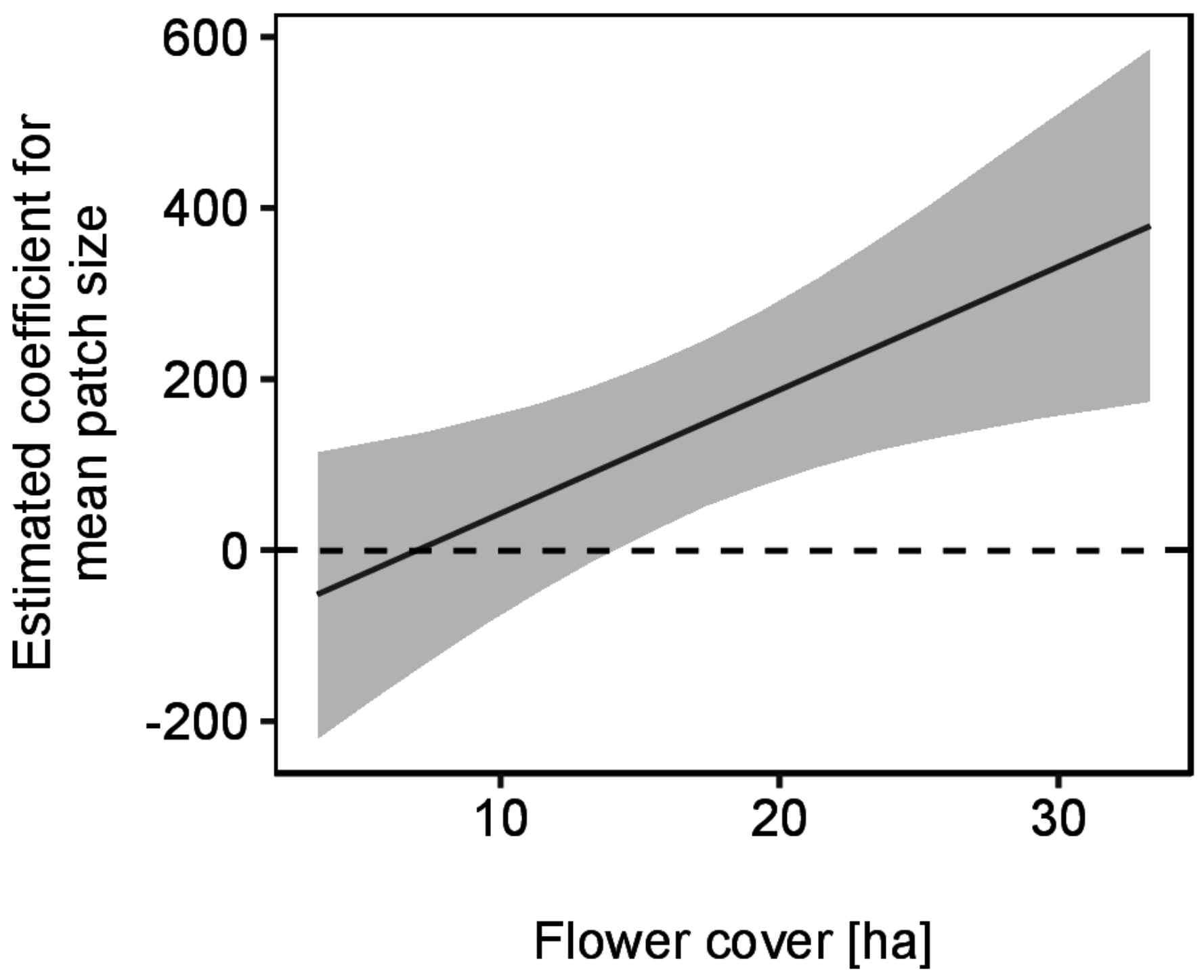

